\title{
Effects of soil profile and characteristic of exciting force on propagation of ground vibrations
}

\author{
Sunao KUNIMATSU ${ }^{*}$, Yoshihiro HIRAO ${ }^{2}$, Yasutoshi KITAMURA ${ }^{3}$ \\ 1. Institute for Geo-Resources and Environment, National Institute of Advanced Industrial Science and Technology, 1-1-1 Higashi, \\ Tsukuba, Ibaraki, 305-8567, Japan \\ 2. Kobayashi Institute of Physical Research, 3-20-41 Higashi-Motomachi, Kokubunji, Tokyo, 185-0020, Japan \\ 3. Construction Engineering Research Institute Foundation, 1-3-10 Turukabuto, Nada-ku, Kobe, Hyogo, 657-0011, Japan
}

\begin{abstract}
Ground-borne vibrations caused by vibration sources such as road traffic and construction exhibit complicated properties during propagation from the vibration source to the inside of a building. In the present paper, a numerical analysis technique for the system of vibration source and propagation path of ground vibration is developed in order to systematically determine the propagation properties of the vibration as part of developing a predictive technique for exposure evaluations by vibrations in three directions at receiving points of vibration in the human body. First, the exciting forces in three directions for input into the numerical computation are inversely-estimated by using the measured acceleration records of the measurement points, which are near the vibration source. The thin-layered element method is used for numerical computation of the ground vibration. Then, the calculation results for the ground vibration obtained by using the estimated exciting force are compared with the measured results, and the influence of the stratified structure of the ground on the exciting force and the propagation properties of the ground vibration are studied. From these results, in a prediction of the ground vibration in three directions, it is emphasized that it is necessary to consider the influence of horizontal exciting force, although attention has been paid to only the vertical exciting force for simulating ground vibration.
\end{abstract}

Key words: ground-borne vibration; vibration source; exciting force; stratified structure; soil profile

C) 2012 JMT. All rights reserved.

\section{Introduction}

$\mathrm{T}$ he vibration regulation law (1976) and noise regulation law (1968) were established in order to deal with the numerous noise and vibration complaints that occurred during the period of high economic growth in Japan. The vibration regulation law targets factories, road traffic, and construction sites as vibration sources. The regulation standard is regulated by the vibration level (frequency-weighted acceleration level, unit: $\mathrm{dB}$ ) in the vertical direction at the site boundary of the vibration source based on the concept of regulating the emission source. It has been over 35 years since the establishment of the vibration regulation law and complaints often occur though the measured vibration level at the

This paper was presented at the 5th International Symposium on Environmental Vibration in Chengdu, China, October 2022,2011 , and recommended by the scientific committee of the symposium to JMT

Received Feb. 15, 2012; revision accepted July. 6, 2012

*Corresponding author. Tel.: +81-29-861-8289, Fax: +81-29-

861-3717, E-mail: s.kunimatsu@aist.go.jp

(C) 2012 JMT. All rights reserved

doi: 10.3969/j.issn.2095-087X.2012.03.002 site boundary satisfies the regulation level [1-6]. On the other hand, the evaluation method and evaluation value, such as noise annoyance, at the place where the human body is exposed to the vibration were considered in ISO (ISO 2631-1:1997 and ISO 2631-2:2003) [7-8]. Accordingly, research to reexamine the future of vibration regulation based on exposure evaluation is being advanced [9].

When the exposure regulation is enforced for buildings, including detached houses, exposure evaluation by vibration in three directions is necessary. In addition, it is important to predict with high accuracy the properties of the vibration source, the propagation properties of the ground vibration, and the transmission properties from the ground to the building. In the present study, a technique by which to grasp both the properties of the vibration source and the propagation properties of the ground vibration is proposed for use along the vibration propagation path from the vibration source to the building. The future goal is to develop a prediction method for exposure evaluations that consider the transmission properties from the ground to the building.

In the present study, exciting forces in three directions for input in numerical computation of the ground 
vibration are estimated inversely using acceleration records at the measurement point (hereinafter referred to as the reference point), which is near the vibration source. The relationship among the stratified structure of the ground, the exciting force, and the propagation properties of the ground vibration is considered based on the calculation results.

\section{Procedure of inverse estimation of excit- ing force}

The thin layered element method [10-21] was used for numerical computation of ground-borne vibration in the present work, and a computer program was constructed based on Kausel [16]. The stratified structure of the ground model was prepared based on the soil boring log of a point near the measurement line. The exciting forces and propagation properties are estimated according to Fig. 1, which shows a summary of the proposed procedure. A schematic diagram of the procedure is also shown in Fig. 1. Each of the steps is described in the following:

Step 1 On the ground modeled into thin layers, unit harmonic exciting forces in three directions acted at the position of the vibration source. For unit harmonic exciting forces $P_{i}(\omega)=1(i=x, y, z)$ acting at the position, the displacement in three directions $A_{i j}(\omega)(i, j=x, y, z)$ is obtained at the reference point and at each measurement point. The displacement $A_{i j}(\omega)$ gives $\boldsymbol{A}$ in the expression of $\boldsymbol{U}=\boldsymbol{A P}$ as follows:

$$
\left[\begin{array}{l}
U_{x}(\omega) \\
U_{y}(\omega) \\
U_{z}(\omega)
\end{array}\right]=\left[\begin{array}{lll}
A_{x x}(\omega) & A_{x y}(\omega) & A_{x z}(\omega) \\
A_{y x}(\omega) & A_{y y}(\omega) & A_{y z}(\omega) \\
A_{z x}(\omega) & A_{z y}(\omega) & A_{z z}(\omega)
\end{array}\right]\left[\begin{array}{l}
P_{x}(\omega) \\
P_{y}(\omega) \\
P_{z}(\omega)
\end{array}\right],
$$

where $\boldsymbol{A}$ is referred to as the complex influence coefficient matrix, which is a square matrix of rank $3 ; U_{i}(\omega)$ and $P_{i}(\omega)(i=x, y, z)$ are complex displacements and exciting forces in three directions, respectively.

Step $2 U_{i}(\omega)(i=x, y, z)$ for three directions is obtained by the Fourier transform of the measured acceleration in three directions at the reference points. $P_{i}(\omega)(i=x, y, z)$ for three directions is calculated inversely by $\boldsymbol{P}=\boldsymbol{A}^{-1} \boldsymbol{U}$ for every frequency, and is expressed in component form as follows:

$$
\left[\begin{array}{l}
P_{x}(\omega) \\
P_{y}(\omega) \\
P_{z}(\omega)
\end{array}\right]=\left[\begin{array}{lll}
A_{x x}(\omega) & A_{x y}(\omega) & A_{x z}(\omega) \\
A_{y x}(\omega) & A_{y y}(\omega) & A_{y z}(\omega) \\
A_{z x}(\omega) & A_{z y}(\omega) & A_{z z}(\omega)
\end{array}\right]^{-1}\left[\begin{array}{l}
U_{x}(\omega) \\
U_{y}(\omega) \\
U_{z}(\omega)
\end{array}\right] .
$$

Step $3 U_{i, n}(\omega)(i=x, y, z)$ of measurement point $n$ obtained using the complex exciting forces $P_{i}(\omega)$, which are estimated inversely using Step 2, is obtained through

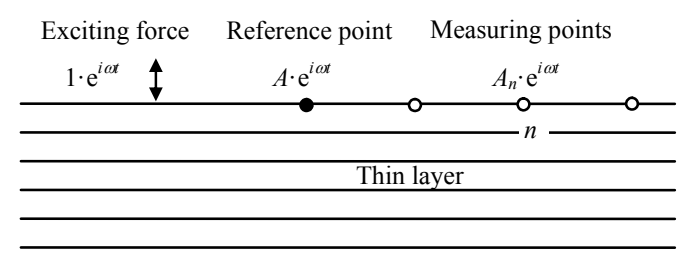

(a) Step 1 (transfer function)

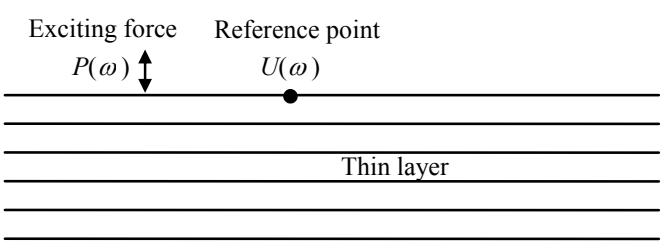

(b) Step 2 (inverse estimation of $P(\omega)$ )

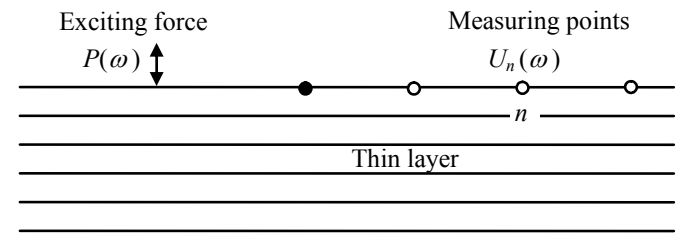

(c) Step 3 (estimation of $U_{n}(\omega)$ )

Fig. 1 Schematic diagram of the procedure of inverse estimation of exciting force

$\boldsymbol{U}=\boldsymbol{A P}$. The displacement $U_{i, n}(\omega)(i=x, y, z)$ is converted into acceleration in the frequency domain.

Based on the Fourier inverse transform of the acceleration, the acceleration waveforms in three directions at the measurement point $n$ are obtained. Next, based on the time history of the accelerations, the vibration acceleration level (VAL) and one-third octave band spectra at the measurement point $n$ are obtained.

\section{Modeling of real ground to stratified structure}

The surveyed vibration sources are four road traffic sites (sites $A, B, C$, and $E$ ) and one construction site (site $D)$. Fig. 2 shows a columnar section of the $\mathrm{S}$ wave velocity $\left(V_{\mathrm{s}}\right)$ for all sites for use in the numerical simulation. Columnar sections of the $\mathrm{S}$ wave velocity are converted from the $\mathrm{N}$-value obtained through a standard penetration test. As an example, columnar sections of the $N$-value and the $\mathrm{S}$ wave velocity are shown for site $B$. In addition, the Poisson's ratio, density, and damping of ground, which are necessary for numerical simulation, are assumed according to geological classification based on various documents.

The $N$-values of sites $A, B, C$, and $E$ are based on survey results along the measurement line, but the $N$ - 

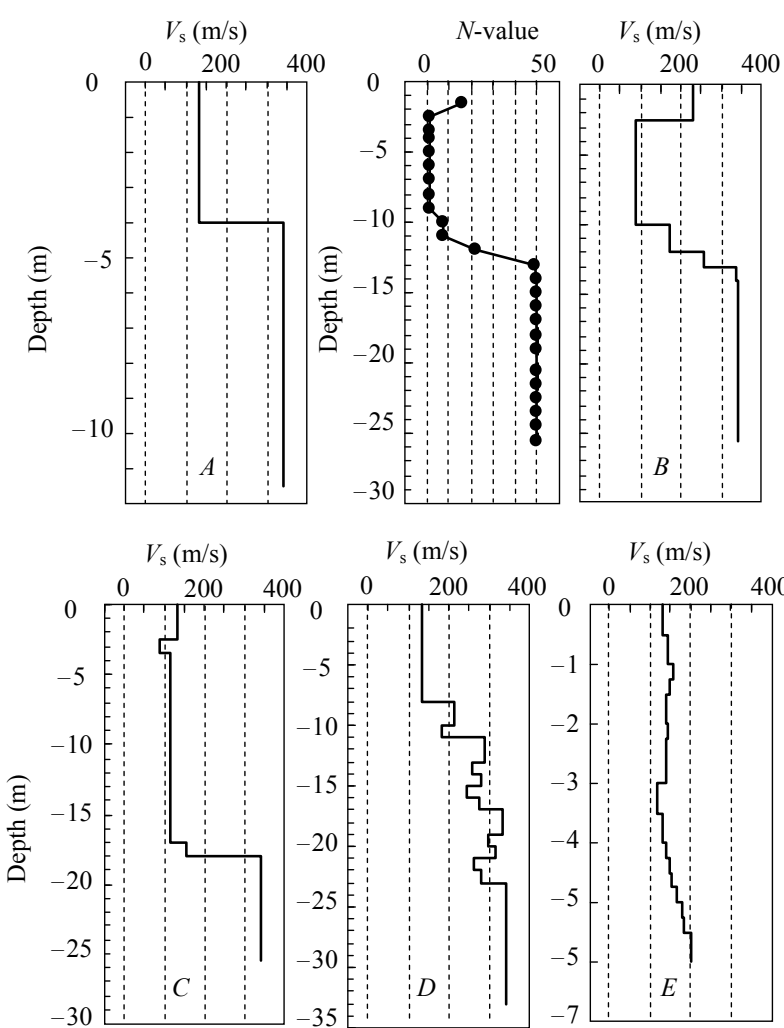

$V_{\mathrm{s}}(\mathrm{m} / \mathrm{s})$
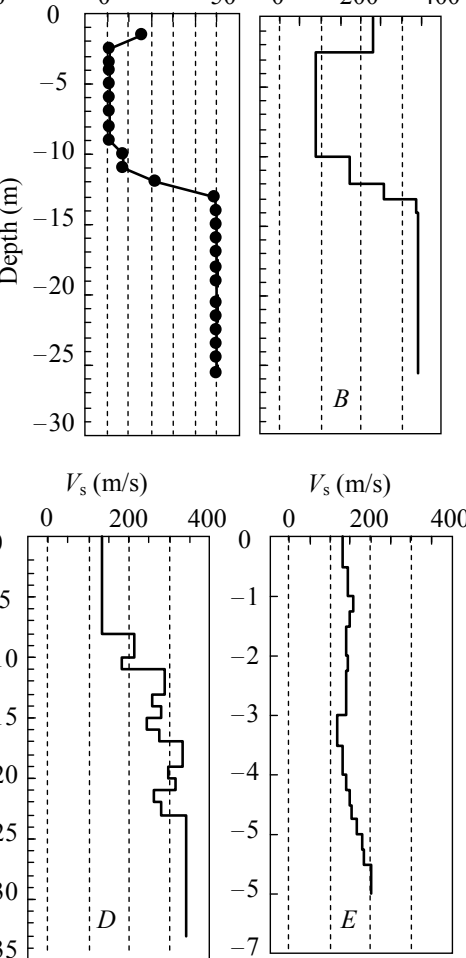

$V_{\mathrm{s}}(\mathrm{m} / \mathrm{s})$

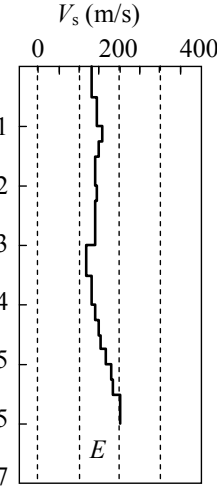

Fig. 2 Columnar section of $\mathrm{S}$ wave velocity for all sites value of site $D$ is based on survey results for the site at a location approximately $570 \mathrm{~m}$ from the measurement line. In addition, sites $A, B$, and $C$ are chosen to examine the influence of the depth of the base layer with $N$ values exceeding 50 and site $E$ is the site that is chosen in order to examine the vibration transmission property from the ground to a building, although the results are not presented herein.

\section{Properties of exciting force by inverse es- timation}

Fig. 3 shows the acceleration waveforms recorded at the reference point of each site. In these figures, the $Y$ direction is the one orthogonal to the road traffic direction and is the direction of the measurement line for construction work. In addition, the distance from the edge of the road is $2.3 \mathrm{~m}$ for sites $A, B$, and $C$ and $9 \mathrm{~m}$ for site $E$; the distance from the vibration source is $10 \mathrm{~m}$ for site $D$.

The frequency spectra of exciting forces $\left(P_{x}, P_{y}, P_{z}\right)$ in three directions that are inversely estimated at each measurement site are shown in Fig. 4 by one-third octave band of frequency spectra, for one-third octave band spectrum is conventionally used to show the frequency spectrum of ground vibration in the field of environmental vibration.
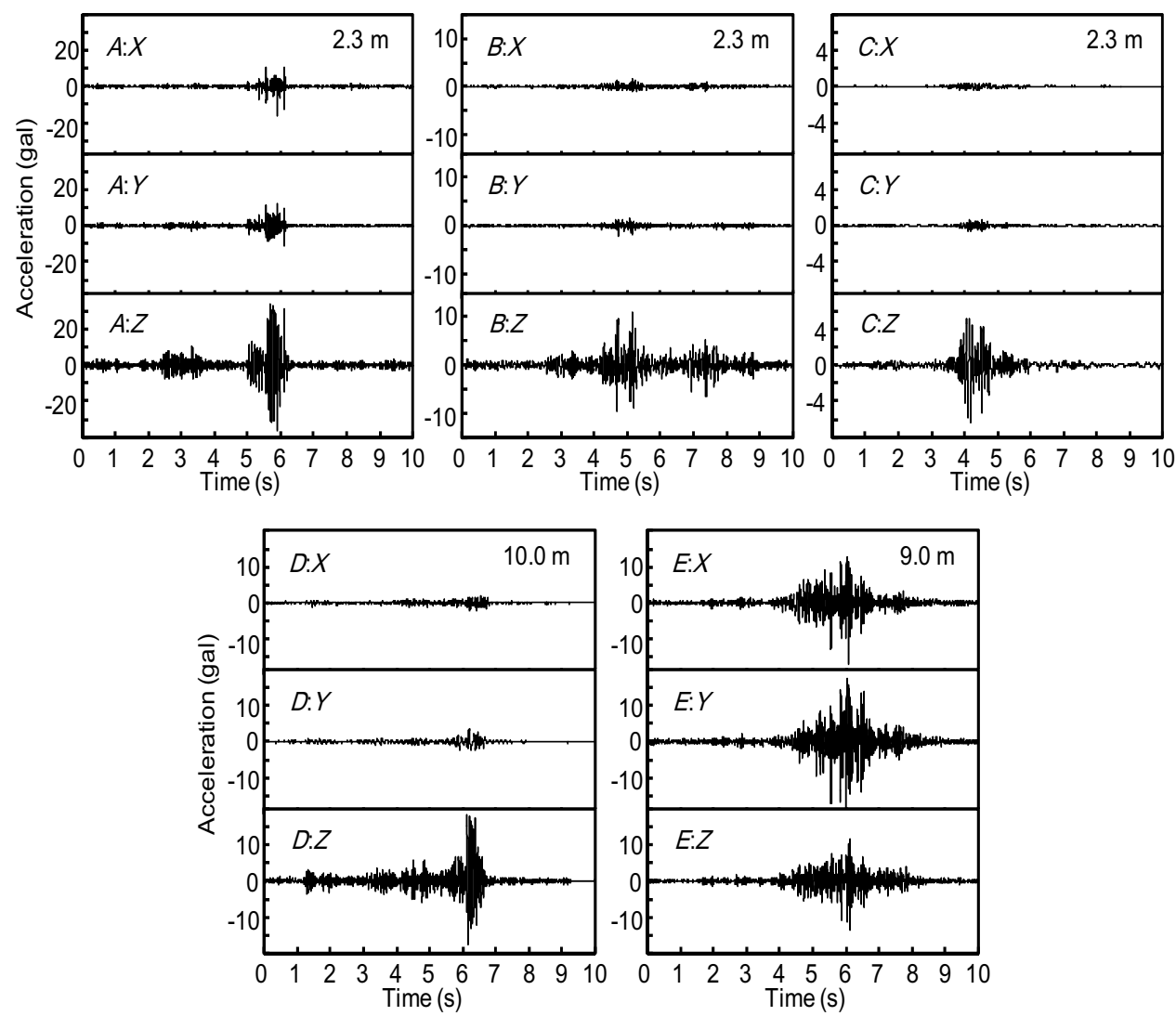

Fig. 3 Acceleration waveforms recorded at reference point of each site 

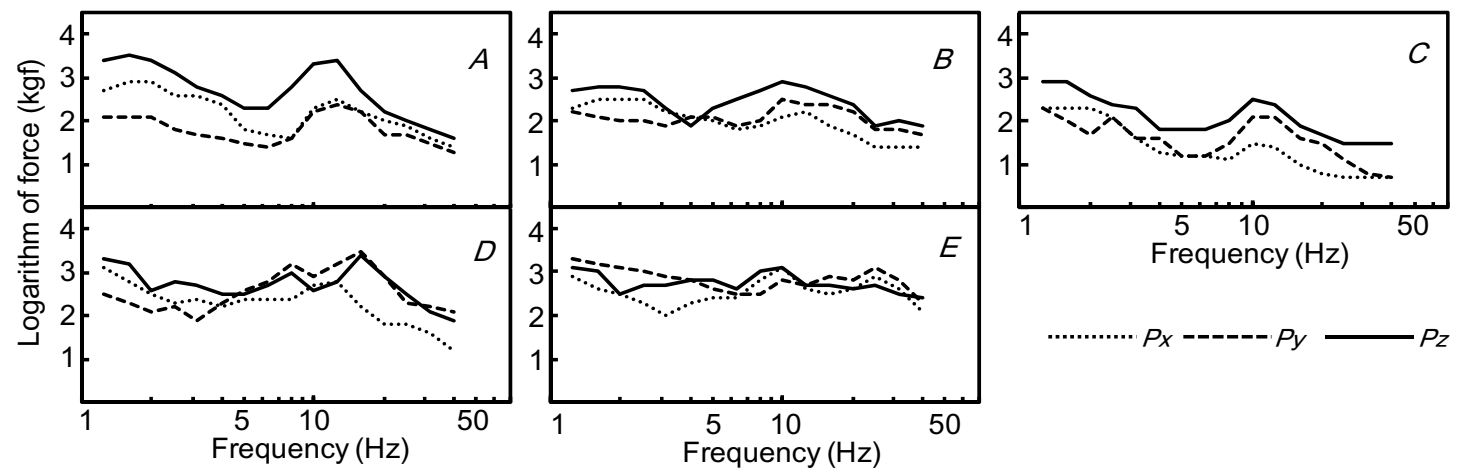

Fig. 4 The spectra of exciting forces in each direction at all sites by inverse estimation

The spectra are compared for sites $A, B$, and $C$, which are chosen in order to examine the influence of the depth of the base layer. The shape of the spectrum for site $C$ resembles that for site $A$, and the peak frequency is also the same. However, the overall spectrum level for site $C$ is smaller than that for site $A$. At site $B$, which has a middle layer, the spectrum shape around the high-frequency peak is different from the shape for other sites. The spectrum level for site $B$ is between those of sites $A$ and $C$. Thus, the stratified structure of the ground is thought to be related to the properties of exciting forces.

At site $E$, the horizontal exciting force is at the same level as the vertical exciting force. In the estimation of road traffic vibration, conventionally, the vertical ground vibration caused by only vertical exciting force has been investigated. However, there is thought to be a need to investigate the influence of horizontal exciting forces because actual displacements are the superposition of displacement by vertical exciting force and displacement by horizontal exciting force. In addition, the horizontal exciting force in the $Y$ direction is at the same level as the vertical exciting force, and the spectrum shapes of these forces are similar, even for the construction work site, i.e., site $D$. In this case, an investigation of the influence of the horizontal exciting force is necessary.

\section{Time history of the acceleration of the ground vibration}

The waveforms of the measured acceleration are compared with those calculated based on the inversely estimated exciting force. There are four or five measurement points, including the reference point, in each site except site $E$ where there are two measurement points, namely, the reference point and a point $44 \mathrm{~m}$ from the source. Here, the results of a comparison of the measured waveform and the calculated waveform at one measurement point are shown in Fig. 5 for each site. Since the measured waveforms of the reference point and of each point are out of synchronization, the time of the calculated waveform is matched with the time of the measured waveform by visual comparison except the reference point.

At site $D$ (the construction site), some differences in amplitude in the $Z$ direction are observed, but the calculated waveform and the measured waveform exhibit good correspondence. With respect to road traffic, the change of waveform in the time history of acceleration reflects the change of distance between moving vibration source and measurement point. Therefore, the amplitude of the waveform sharply becomes large as the moving vibration source approaches the measurement point near the road. In contrast, the change of the amplitude in the waveform becomes small as the distance between the vibration source and the measurement point increases with the distance from the road. The calculation results show the expected phenomenon.

At sites $A, B$, and $C$, the waveform with the large amplitude is mixed with the measured waveform except the waveform that is assumed according to the target vehicle (a vibration source). At site $B$, the vibration generated by the following vehicle is observed after approximately $5 \mathrm{~s}$, although the vibration is not observed in the measured waveform at the reference point in Fig. 3. In addition, the waveform for which the amplitude is recognized to be relatively large and a low-frequency component are mixed with the measured waveform. By dissociating these mixed components from the measured waveform, it seems that the appearance of the wave patterns of both the calculated waveform and the measured waveform is roughly equivalent.

As the distance between the measurement point from the road increases, the calculated waveform does not generally coincide with the measured one, because the influence of background vibration becomes relatively larger in the measured vibration. Although the measurement point is far from a road, for site $E$, as for the calculated waveform and the measured waveform, the appearance of the wave patterns is roughly equivalent although a difference is observed in the amplitude. 

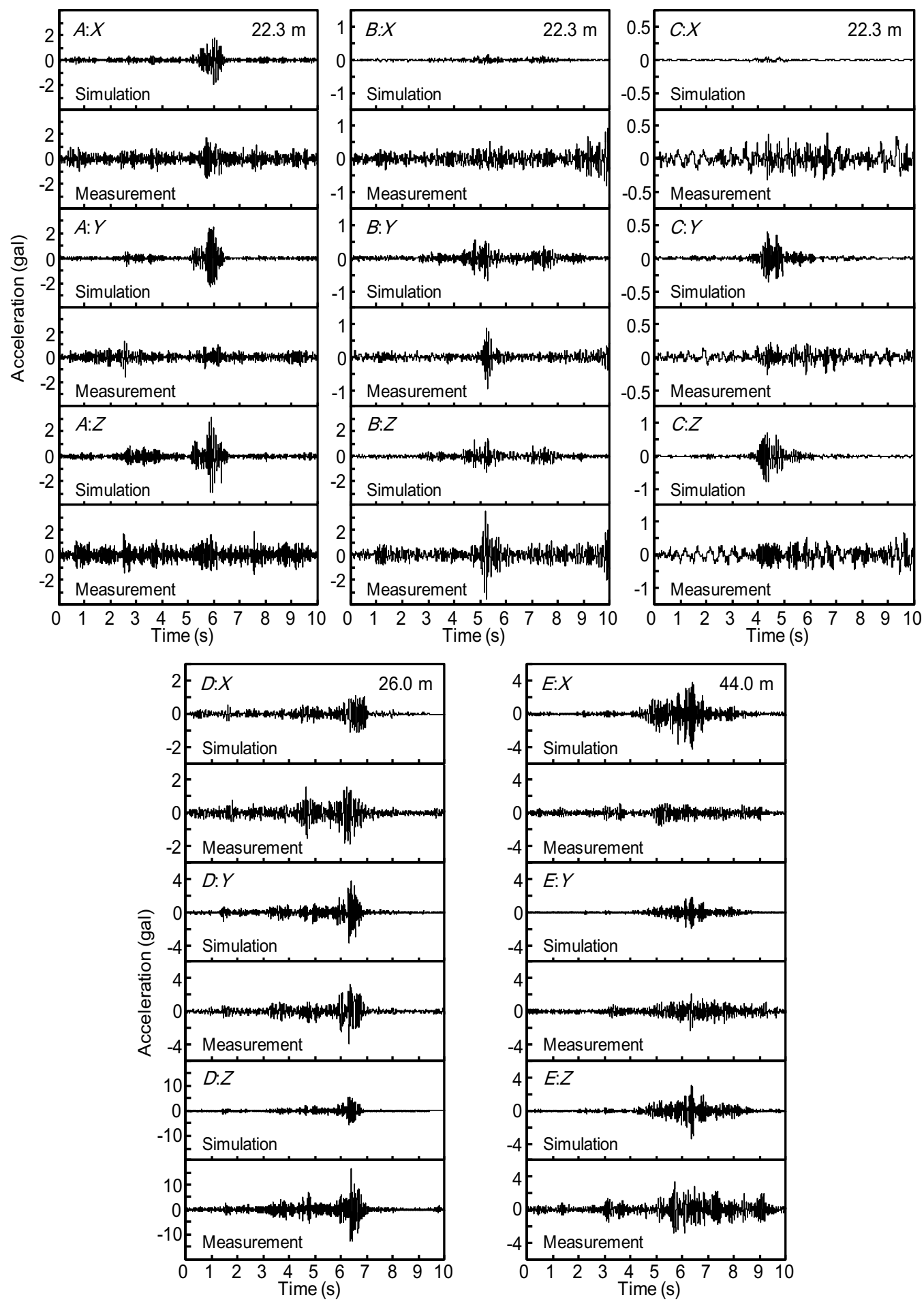

Fig. 5 The comparison of the calculated wave form and the measured wave form in one measuring point of each site

Although, for road traffic vibration, the difference between the calculated waveform and the measured waveform is not necessarily small, aside from the technique proposed herein, a useful method for estimating the exciting force of the vibration source has not yet been found [22-34]. In the future, it will be necessary to examine the effectiveness of the proposed method by in- creasing the types of applications. In the examination, the vibration generated by an independent vehicle must be measured without being influenced by other vehicles.

In addition, the validity of this technique in the frequency domain is clarified through the comparison between the calculated and measured results in the frequency analysis described in the next section. 


\section{Frequency analysis of the acceleration of the ground vibration}

The establishment of a predictive technique for the exposure evaluation that reflects the frequency properties of a vibration source and the ground propagation is desirable, as noted in the introduction when a shift from the current emission regulation to the future exposure regulation is considered. Since a grasp of frequency properties is important with respect to the frequency properties of the vibration source changing in a process propagating through the ground, the ground vibration does not attenuate monotonically. In addition, the prediction result can be utilized for vibration reduction measures. In the field of environmental vibration, since one-third octave band spectra are used in frequency analysis of measured vibrations more frequently than FFT spectra, the frequency properties of the calculated vibrations obtained by the predictive method are also examined by one-third octave band spectra.

At each site, the one-third octave spectrum of the measured acceleration is compared with the calculated one by the exciting force estimated inversely in Figs 6 to 10. The vertical axis shows the vibration acceleration levels of the decibel unit. The calculated spectra for ground models M1 and M2 are shown in these figures. Model M1 simulates the real ground structure using the stratified structure based on a soil columnar section, and model M2 simulates the real ground structure based on the half-infinity ground with the same ground constant as the surface layer.

As a matter of course, the measured spectrum (black dots in the figure) agrees with the calculated spectrum
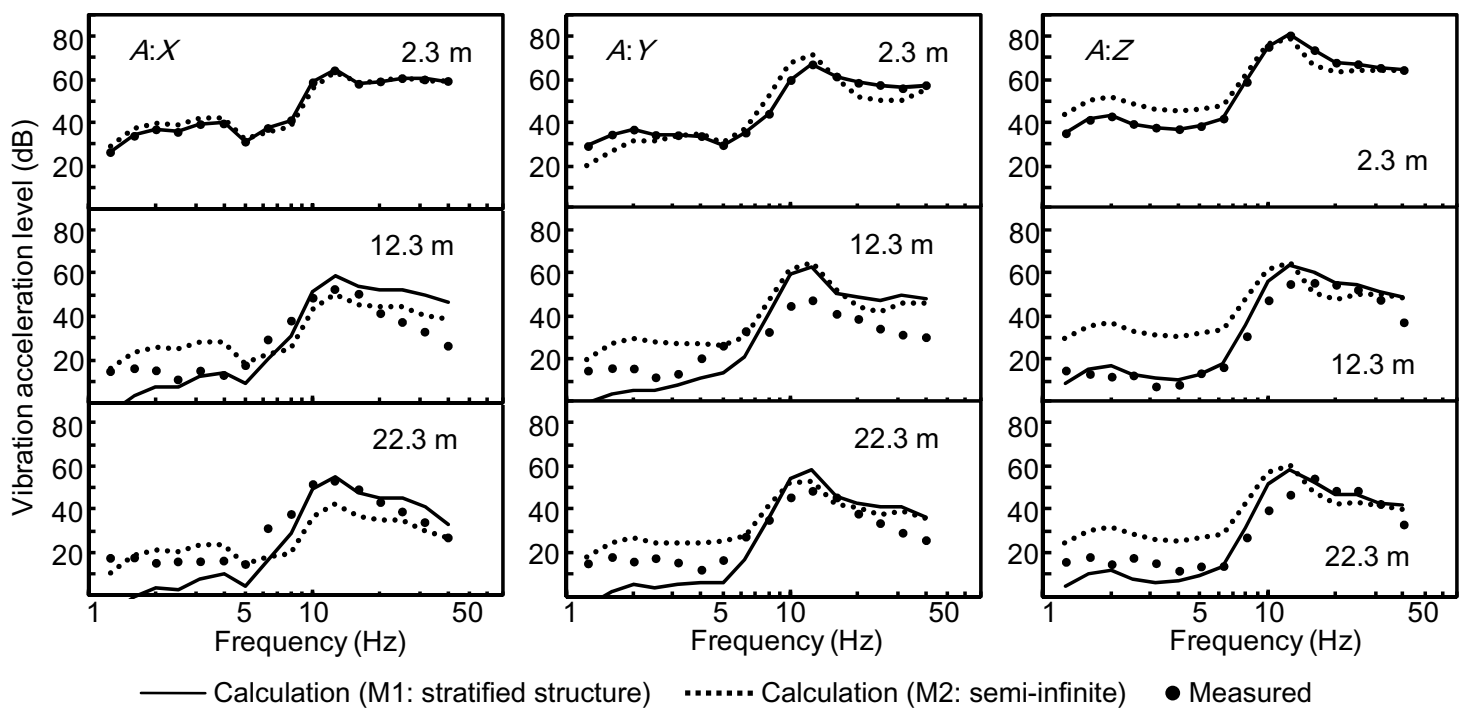

Fig. 6 The results of one-third octave band analysis at several points in site $A$
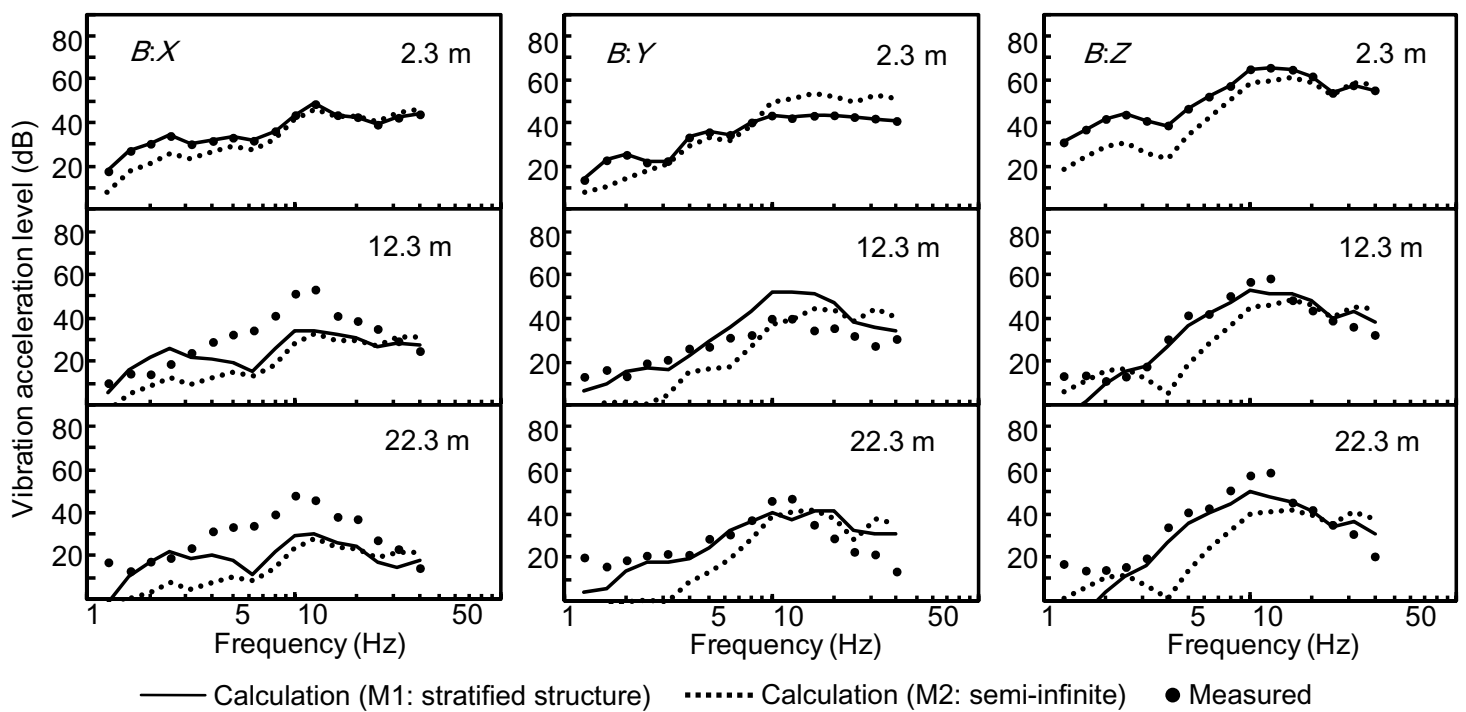

Fig. 7 The results of one-third octave band analysis at several points in site $B$ 
(solid lines in the figure) of model $\mathrm{M} 1$ in $2.3 \mathrm{~m}$ point for sites $A, B$, and $C, 10 \mathrm{~m}$ point of site $D$, and $9 \mathrm{~m}$ point of site $\mathrm{E}$ because these points are the reference points used to estimate exciting forces in each site.

The calculated spectrum for model $\mathrm{M} 1$ at sites $A, D$, and $E$ matches the measured spectrum, but the difference between the calculated and measured spectra appears as the distance increases and this difference is remarkable in the $X$ direction. The difference of frequency spectra between the calculated vibration and the measured vibration is not directly reflected by the difference of waveform between the calculated waveform and the measured waveform shown in Fig. 5, because the onethird octave band spectrum is the peak-hold level of the vibration acceleration level of the vibration acceleration passed in each band.
Next, the spectra of models M1 and M2 agree fairly well for the spectra calculated for sites $C, D$, and $E$. On the other hand, at site $A$, the high-frequency components of these models agree, but there is a difference in the low-frequency components (a long wavelength). When the base layer is deep, as in the cases of sites $C$ and $D$ or when the stratified structure of the ground is almost uniform, as in the case of half-infinity ground, such as that at site $E$, the ground is considered to be a half-infinity medium. Therefore, the depth is thought to influence the difference in the spectrum at low frequencies in the shallow site $A$ of the base layer. At site $B$, a difference in spectrum shape is observed for models M1 and M2 at low frequency. This is considered to be due to the influence of the intermediate layer of the low $N$-value level.
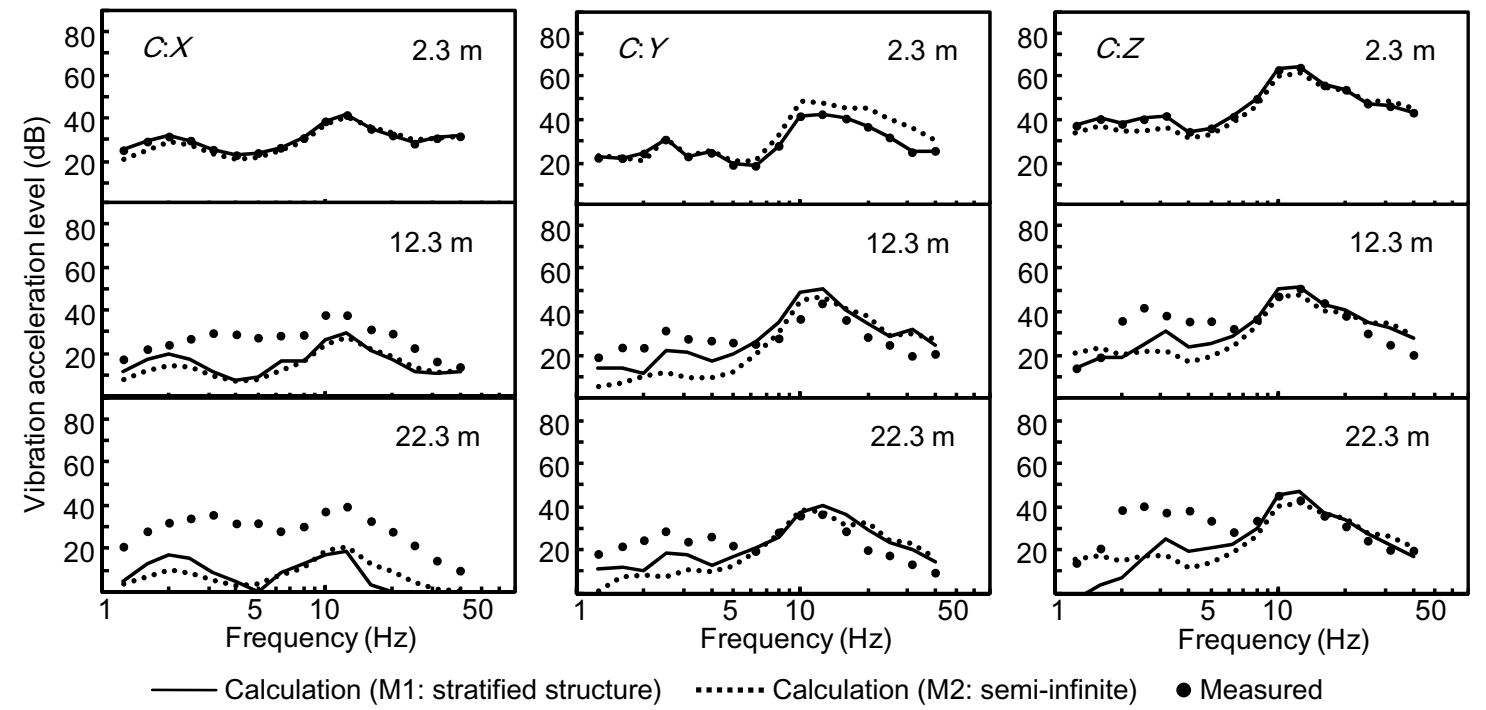

Fig. 8 The results of one-third octave band analysis at several points in site $C$
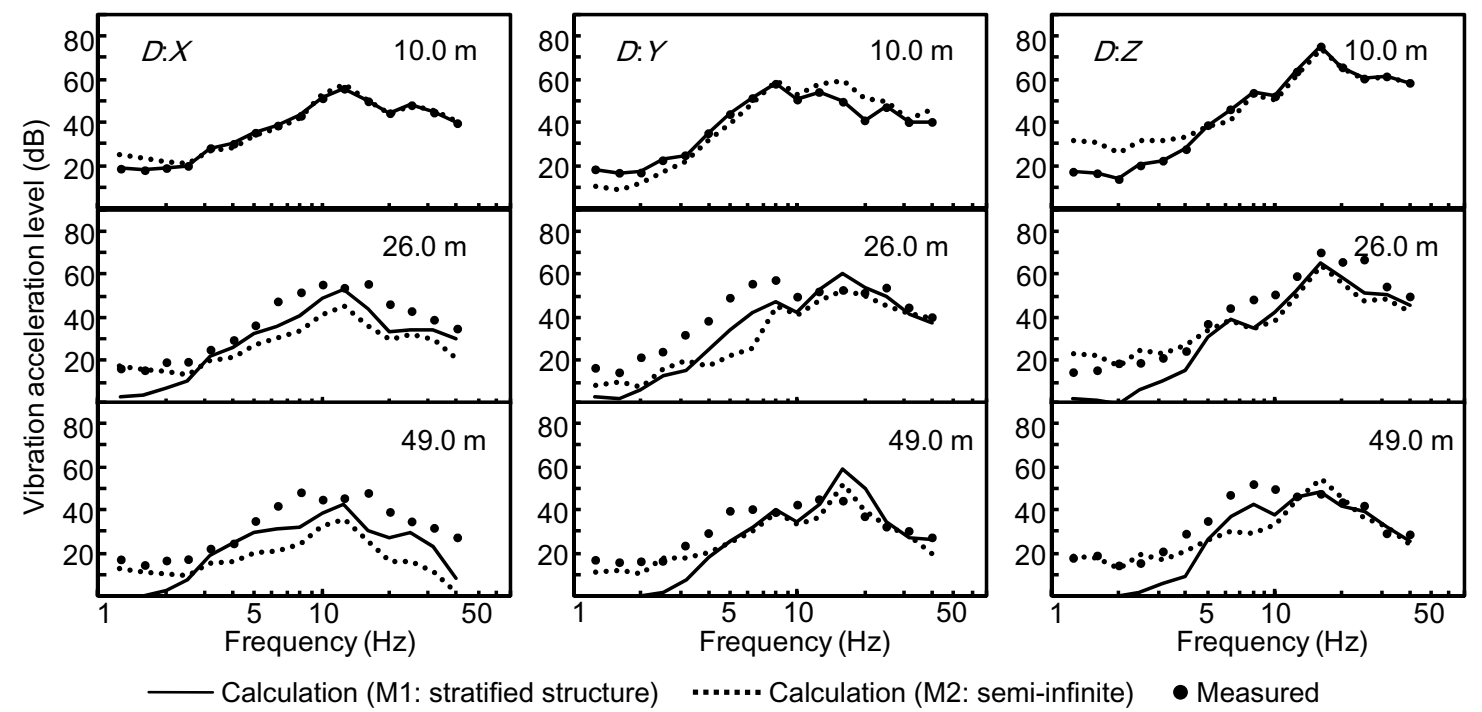

Fig. 9 The results of one-third octave band analysis at several points in site $D$ 

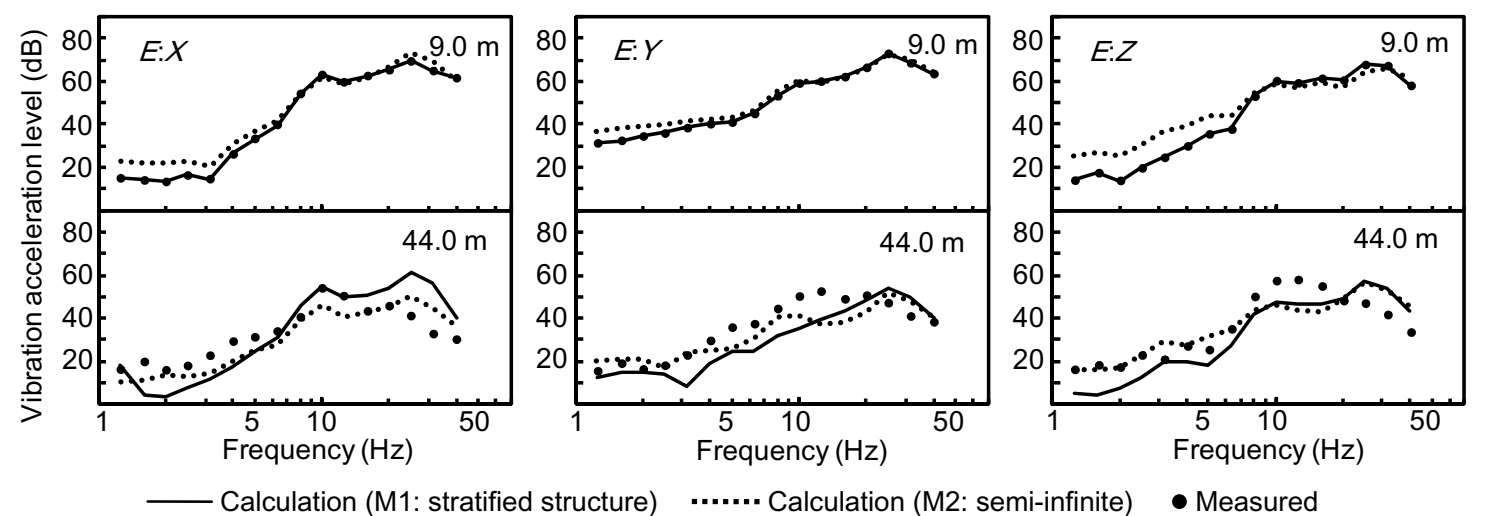

Fig. 10 The results of one-third octave band analysis at several points in site $E$

\section{Conclusions}

In the present paper, a method by which to estimate the exciting force inversely using the measured acceleration record at a point near the vibration source was proposed. The results calculated with the use of the exciting forces are compared with the data measured at several sites, and good agreement is identified for the waveforms and frequency analysis. Although it will be necessary in the future to inspect the effectiveness of the proposed method by increasing the types of applications, the proposed predictive technique is thought to be a useful method because a more useful method has not yet been found under the present conditions. In addition, the horizontal exciting force should be considered in the prediction of the ground vibration of three directions. Furthermore, in the future it will be necessary to develop a predictive technique for exposure evaluations by incorporating the transmission properties from the ground to the building.

\section{Acknowledgements}

The present study was supported in part by the Ministry of the Environment of Japan.

\section{References}

[1] J. Yue, The movement and subject of the vibration administration, In: Proc. of the 2004 spring meeting of INCE/J, Tokyo, Japan, 2004: 5-8 (in Japanese).

[2] Ministry of the Environment, Tokyo, Japan, Review of the execution status of the Vibration Regulation Law in the year Heisei 16, 2005 (in Japanese).

[3] K. Takemoto, K. Naito, M. Fujimoto, et al., The Present Situation and the Subject of Environmental Vibration in Japan, In: Proc. of INTER-NOISE 2006 (CD-ROM), Hawaii, USA, 2006: 1-7.

[4] Y. Matsumoto, S. Kunimatsu, Y. Hirao, et al., Vibration measurement in residential building for evaluation of ground-borne vibration in living environment, In: Proc. of INTER-NOISE 2006 (CD-ROM), Hawaii, USA, 2006: 1-10.

[5] K. Takemoto, K. Naito, Y. Yamashita, et al., Recent action of MOE for environmental vibration in Japan, In: Proc. of Int. Symposium on Environmental Vibration, , Taipei, Taiwan, 2007: 139-147.

[6] K. Nagamori, Current conditions and issues about administrative control for vibration as environmental pollution, In: Proc. of the 2009 spring meeting of INCE/J, Tokyo, Japan, 2009: 33-36 (in Japanese).

[7] International Standard ISO 2631-1: 1997, Mechanical vibration and shock - Evaluation of human exposure to whole-body vibration - Part 1: General requirements, International Organization for Standardization, Geneva, Switzerland, 1997.

[8] International Standard ISO 2631-2: 2003, Mechanical vibration and shock-Evaluation of human exposure to whole-body vibration-Part 2: Vibration in buildings (1 $\mathrm{Hz}$ to $80 \mathrm{~Hz}$ ), International Organization for Standardization, Geneva, Switzerland, 2003.

[9] S. Kunimatsu, H. Hirao, Vibration characteristics of house in Japan by $1 / 3$ oct. band frequency to environmental vibration estimation, In: 4th International Symposium on Environmental Vibration, Beijing, Oct.28-30, 2009: 134-139.

[10] J. Lysmer, G. Waas, Shear waves in plane infinite structures, Journal of Engineering Mechanics, 1972, 98(1): 85-105.

[11] H. Tajimi, Y. Shimomura, Approximate solver of the vibration of foundation-soil interaction by the layer segmentation, In: Proc. of Annual Convention of AIJ, Fukuoka, Japan, 1972: 485-486.

[12] E. Kausel, J.M. Roesset, Dynamic stiffness of circular foundation, Journal of Engineering Mechanics, 1975, 101(6): 771-785.

[13] H. Tajimi, Y. Shimomura, Dynamic analysis of soilstructure interaction by the thin layered element method, Transactions of the Architectural Institute of Japan, 1976, 243: 41-51.

[14] H. Tajimi, A contribution to theoretical prediction of dynamic stiffness of surface foundation, In: Proc. of 7th 
World Conference Earthquake Engineering, Istanbul, Turkey, 1980, 5:105-112.

[15] G. Waas, Dynamically-loaded foundation on the stratified ground, VDI-Berichte, 1980, 381: 185-189 (in German).

[16] E. Kausel, An explicit solution for the Green functions for dynamic loads in layered media, Research Report R81-13, No.699, MIT, 1981: 1-79.

[17] S. Takano, Y. Yasui, M. Iguchi, Point load solutions on layered fluid-filled poroelastic soil by thin layered element method and its application, Journal of Structural and Construction Engineering, AIJ, 1998, 504: 49-56 (in Japanese).

[18] S. Takano, Y. Yasui, M. Iguchi, Dynamic soil-structure interaction analysis of single piles in layered fluid-filled poroelastic soil by thin layered element method, Journal of Structural and Construction Engineering, AIJ, 1998, 512: 91-98 (in Japanese).

[19] S. Takano, Y. Yasui, M. Iguchi, Dynamic soil-structure interaction analysis of pile groups in layered fluid-filled poroelastic soil by thin layered element method, Journal of Structural and Construction Engineering, AIJ, 1999, 515: 67-74 (in Japanese).

[20] T. Jiang, H. Tajimi, Analytical procedure of SoilStructure-Interaction, Shanghai: Tongji University Press, 2000 (in Chinese).

[21] N. Taguchi, T. Hanazato, Y. Ikeda, et al., Analysis method combining finite element method with thin layer method and its application, Journal of Environmental Engineering, AIJ, 2008, 626: 423-429 (in Japanese).

[22] M. Gunaratne, O. Sanders, Response of a layered elastic medium to a moving strip load, Int. Journal for Numerical and Analytical Methods in Geomechanics, 1996, 20 : 191-208.

[23] Y. Kitamura, S. Takesue, S. Sumitomo, Simulation and observations of ground vibration due to a moving traffic excitation, In: Proc. of INTER-NOISE 1998 (CD-ROM), Christchurch, New Zealand, 1998: 1-7.

[24] G. Lombaert, G. Degrande, D. Clouteau, Numerical modelling of free field traffic-induced vibrations, Soil Dynamics and Earthquake Engineering, 2000, 19: 473488.
[25] H.H. Hung, Y.B. Yang, Elastic waves in visco-elastic half-space generated by various vehicle loads, Soil $D y$ namics and Earthquake Engineering, 2001, 20: 1-17.

[26] H. Hirose, Efficient numerical approach for the analysis of vibrations due to moving load, In: Int. Workshop Wave 2002 on Wave Propagation, Moving Load and Vibration Reduction, Okayama, Japan, 2002: 113-119.

[27] H. Pezeshki, Ground vibrations in the vicinity of multiple structures on a layered media due to waves radiated by an adjacent source [Dissertation], Kobe: Kobe University, 2003.

[28] H. Takemiya, M. Kojima, 2.5-D FEM simulation for vibration prediction and mitigation of track and ground interaction under high-speed trains, In: Proc. of Int. Seminar on Environmental Vibration, Hangzhou, China, 2003: 130-138.

[29] Y. Kitamura, Simulation and observations of ground vibration caused by road traffic, Environmental Vibrations, China Communications Press, 2003: 202-216.

[30] Y. Kitamura, K. Yamamoto, T. Ohguni, A study on a simulation method of traffic-induced ground vibration based on moving test of heavy vehicle, Memoirs of Construction Engineering Research Institute Foundation, 2005, 47: 1-17.

[31] T. Nishimura, K. Hayakawa, Effectiveness of numerical simulation in the prediction of ground vibration problems, In: Proc. of inter-noise 2008 (CD-ROM), Shanghai, China, 2008: 1-7.

[32] N. Taguchi, T. Hanazato, Y. Ikeda, et al., Dynamic loads of traffic-induced ground vibrations on load, Journal of Environmental Engineering, AIJ, 2008, 633: 1241-1247 (in Japanese).

[33] S. Takano, F. Sasaki, Vibration effect of demolish work on adjacent building, In: Proc. of Annual Convention of AIJ, Toyama, Japan, 2010: 403-404 (in Japanese).

[34] Y. Yamada, F. Sasaki, K. Iiyama, et al., A study on the environmental vibration affecting the neighboring ground and building-Calculation of three dimension vibration force in work of construction machine, In: Proc. of Annual Convention of AIJ, Toyama, Japan, 2010: 405406 (in Japanese).

(Editor: Junsi LAN) 